\title{
Including a Priori Knowledge in the Solution of the Inverse Problem During Atrial Fibrillation
}

\author{
Víctor Suárez-Gutiérrez ${ }^{1}$, Miguel Ángel Cámara ${ }^{1}$, Óscar Barquero-Pérez ${ }^{1}$, Ismael Hernández ${ }^{1,2}$, \\ María S. Guillem ${ }^{2}$, Andreu M. Climent ${ }^{3}$, Felipe Alonso-Atienza ${ }^{1}$, Carlos Figuera ${ }^{1}$. \\ ${ }^{1}$ Dept. of Signal Theory and Communications, Universidad Rey Juan Carlos, Madrid, Spain \\ ${ }^{2}$ ITACA, Universitat Politècnica de València, València, Spain \\ ${ }^{3}$ Instituto de Investigación Sanitaria, Hospital Gregorio Marañón, Madrid, Spain
}

\begin{abstract}
Regularization methods for the inverse problem face important challenges during atrial fibrillation $(A F)$, so any a priori information available during the electrophysiological study may improve the solution. We propose a Tikhonov-based inverse problem formulation that incorporates extra information provided by noisy intracavitary measurements near the endocardium. Since we introduced two different sources of information (Tikhonov estimation and a priori measurements), two regularization parameters must be tuned using a generalized L-Curve method. Performance of this method is studied using several timebased metrics proposed in previous works. The method proposed improved estimations accuracy in sinus rhythm, simple and complex AF models.
\end{abstract}

\section{Introduction}

Atrial fibrillation (AF) is the most common type of arrhythmia found in clinical practice, and it affects more than 33 million patients in the world [2]. Furthermore, this pathology is associated to an increased risk of embolism, cardiac failure and mortality [3]. To restore sinus rhythm, ablation techniques based on the isolation of AF sources have been proposed as a successful approach [4], and several works have developed new strategies for locating these AF drivers [5,6].

Due to the spatio-temporal limitations of classical ECG, electrocardiographic imaging (ECGI) allows to effectively reconstruct the electrophysiological activity on the heart surface from non-invasive-recorded body surface potentials (BSP) [7,8]. However, the inverse problem in ECGI is ill-posed, and it is necessary to apply regularization methods to obtain realistic solutions $[9,10]$. Our hypothesis is that any a priori information available during the electrophysiological study may improve the estimation of electrical activity on the heart. In this work, we propose a
Tikhonov-based inverse problem formulation that incorporates noisy intracavitary measurements near endocardium, in order to compare its performance with BSP classical Tikhonov regularization techniques.

The remaining of the paper is organized as follows. In Section 2.1 we introduce the computational model used for this study, the Tikhonov-based regularization techniques, and the performance metrics. Final results are summarized in Section 3 and ins Section 4 main conclusions are presented.

\section{Methods}

\subsection{Computerized Models}

Forward problem is simulated using realistic computational models of both atria ( $\mathrm{N}=2039$ nodes) and torso ( $\mathrm{M}=659$ nodes), to simulate the associated BSP using boundary element method. Sinus rhythm and AF patterns are both simulated, from which the epicardial distribution of potentials are calculated applying Tikhonov-based regularization methods. Simulated BSP are referenced to the Wilson Terminal Center, corrupted with additive Gaussian noise $(\mathrm{SNR}=20 \mathrm{~dB})$ and filtered using a $4^{\text {th }}$-order bandpass Butterworth filter $\left(\mathrm{fc}_{1}=3 \mathrm{~Hz}\right.$ and $\mathrm{fc}_{2}=30 \mathrm{~Hz}$ for $\mathrm{AF}$ models, $\mathrm{fc}_{1}=0 \mathrm{~Hz}$ and $\mathrm{fc}_{2}=30 \mathrm{~Hz}$ for SR) $[1,11]$.

Three propagation patterns are considered:

- Normal sinus rhythm (SR): atrial tissue activated at 1.2 $\mathrm{Hz}$.

- Simple AF propagation pattern (SAF): represented by a right-to-left dominant frequency gradient, with a single functional re-entry located in the right atria which rotates at $7.3 \mathrm{~Hz}$. The rest of the atrial tissue is activated at $4.7 \mathrm{~Hz}$. - Complex AF propagation pattern (CAF): with $25 \%$ of atrial cells under fibrotic conditions [6] and a single functional re-entry located near the right superior pulmonary vein (RSPV) at $6.8 \mathrm{~Hz}$. The rest of the atrial tissue is activated at $5.4 \mathrm{~Hz}$. 


\subsection{Inverse Problem}

Epicardial potentials are estimated using regularization methods with Tikhonov-based formulations. We assume the linear model $\boldsymbol{y}_{t}=\boldsymbol{A} \boldsymbol{x}_{t}+\boldsymbol{\epsilon}$ where $\boldsymbol{\epsilon}$ represents the model residuals. The objective is to estimate epicardial potentials $\mathbf{x}_{\mathbf{t}}$ from BSP, using matrix $\mathbf{A}$. The Tikhonovbased methods analyzed in this work are the following.

Classical Tikhonov regularization (Tikh). It is usually employed in linear inverse problems to stabilize the solution by penalizing its complexity. To obtain the epicardial potentials $\boldsymbol{x}_{t}$ at instant $t$, the functional to minimize is:

$$
\left\|\boldsymbol{y}_{t}-\boldsymbol{A} \boldsymbol{x}_{t}\right\|_{2}^{2}+\lambda_{1}\left\|\boldsymbol{L} \boldsymbol{x}_{t}\right\|_{2}^{2}
$$

where $\boldsymbol{y}_{t}$ is the vector containing the torso measurements, and $\lambda_{1}$ is the global regularization parameter, which is computed by using the L-Curve method for the totality of time instants [1]. The solution for this problem is:

$$
\hat{\boldsymbol{x}}_{t}=\left(\boldsymbol{A}^{T} \boldsymbol{A}+\lambda_{1} \boldsymbol{L}\right)^{-1} \boldsymbol{A}^{T} \boldsymbol{y}_{t}
$$

$\boldsymbol{L}$ matrix can take the form of an identity matrix (zeroorder Tikhonov), Gradient operator (first-order Tikhonov) and Laplacian operator (second-order Tikhonov).

Constrained Tikhonov regularization (Cons-Tikh). In this work, we propose to incorporate measurements near the endocardium as a constraint in the Tikhonov formulation. Measurementes near the endocardium are recorded using two 64-poles intracavitary catheters:

$$
\left\|\boldsymbol{y}_{t}-\boldsymbol{A} \boldsymbol{x}_{t}\right\|_{2}^{2}+\lambda_{1}\left\|\boldsymbol{L} \boldsymbol{x}_{t}\right\|_{2}^{2}+\lambda_{2}\left\|\boldsymbol{B} \boldsymbol{x}_{r e f}-\boldsymbol{D} \boldsymbol{x}_{t}\right\|_{2}^{2}
$$

that forces the solution in the nodes indicated by diagonal matrix $D$ to be similar (a controlled error is allowed) to the measured potentials $\boldsymbol{x}_{\boldsymbol{r} \text { ef }}$ observed with a measurement matrix $\boldsymbol{B}$. $\boldsymbol{L}$ matrix also indicates the order of the Tikhonov method. The closed-form solution for this formulation is:

$\hat{\boldsymbol{x}}_{t}=\left(\boldsymbol{A}^{T} \boldsymbol{A}+\lambda_{1} \boldsymbol{L}^{T} \boldsymbol{L}+\lambda_{2} \boldsymbol{D}^{T} \boldsymbol{D}\right)^{-1}\left(\boldsymbol{A}^{T} \boldsymbol{y}_{t}+\lambda_{2} \boldsymbol{D}^{T} \boldsymbol{x}_{r e f}\right)$

Processed $\boldsymbol{x}_{\boldsymbol{r} \text { ef }}$ signals are also corrupted with additive Gaussian noise $(\mathrm{SNR}=10 \mathrm{~dB})$, and results are compared with clean $\boldsymbol{x}_{\boldsymbol{r} \text { ef }}$ signals. Regularization parameters are chosen using the L-Hypersurface method [12].

\subsection{Performance metrics}

To assess the performance of regularization methods, we propose several metrics, similarly to those proposed in $[1,13]$. Similarity between real and estimated $\hat{\boldsymbol{x}}$ epicardial potentials is quantified by the relative difference measurement star (RDMS), Pearson's correlation coefficient (CC), and root mean squared error (RMSE). RDMS is computed as:

$$
R D M S=\sqrt{\sum_{k}\left(\frac{x_{k}}{\left\|\boldsymbol{x}^{2}\right\|}-\frac{\hat{x}_{k}}{\left\|\hat{\boldsymbol{x}}^{2}\right\|}\right)^{2}}
$$

\section{Results}

Due to the low differences between clean and corrupted $\boldsymbol{x}_{\boldsymbol{r} \text { ef }}$ signals, results are shown without additive noise. Figure 1 shows the performance of each tested method with SR, SAF and CAF models. Best results are obtained with first-order Cons-Tikh method. There are statistically significant differences between same-order Tikh and Cons-Tikh methods $(p<0.001)$ except for CC in zero-order Tikhonov-based methods. These differences are favourable to Cons-Tikh, with a great improvement in first and second-order methods, obtaining CC values of 0.9 for SR models, see Table 1. Estimations were also improved for SAF and CAF models. Same conclusions are obtained for the rest of SNR values. Finally, differences between 1st-order and 2nd-order Cons-Tikh methods were not significant, while dispersion is higher in 2nd-order.

Figure 2 shows epicardial signal estimations for two different nodes. Left panel shows that epicardial potentials reconstructed using first-order Cons-Tikh methods were able to reproduce high frequency components of the original signal, avoiding low-pass filtering behaviour of classical Tikhonov approach. Right panel shows nodes where none
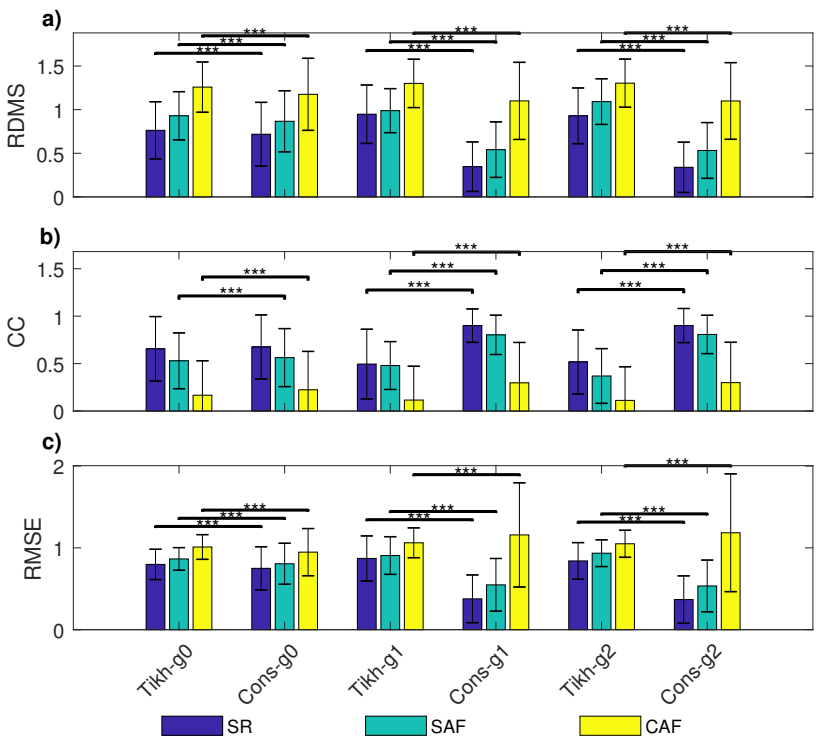

Figure 1. Comparison of RDMS (a), CC (b) and RMSE (c) between different Tikhonov-based estimation methods with SR, SAF and CAF models. 

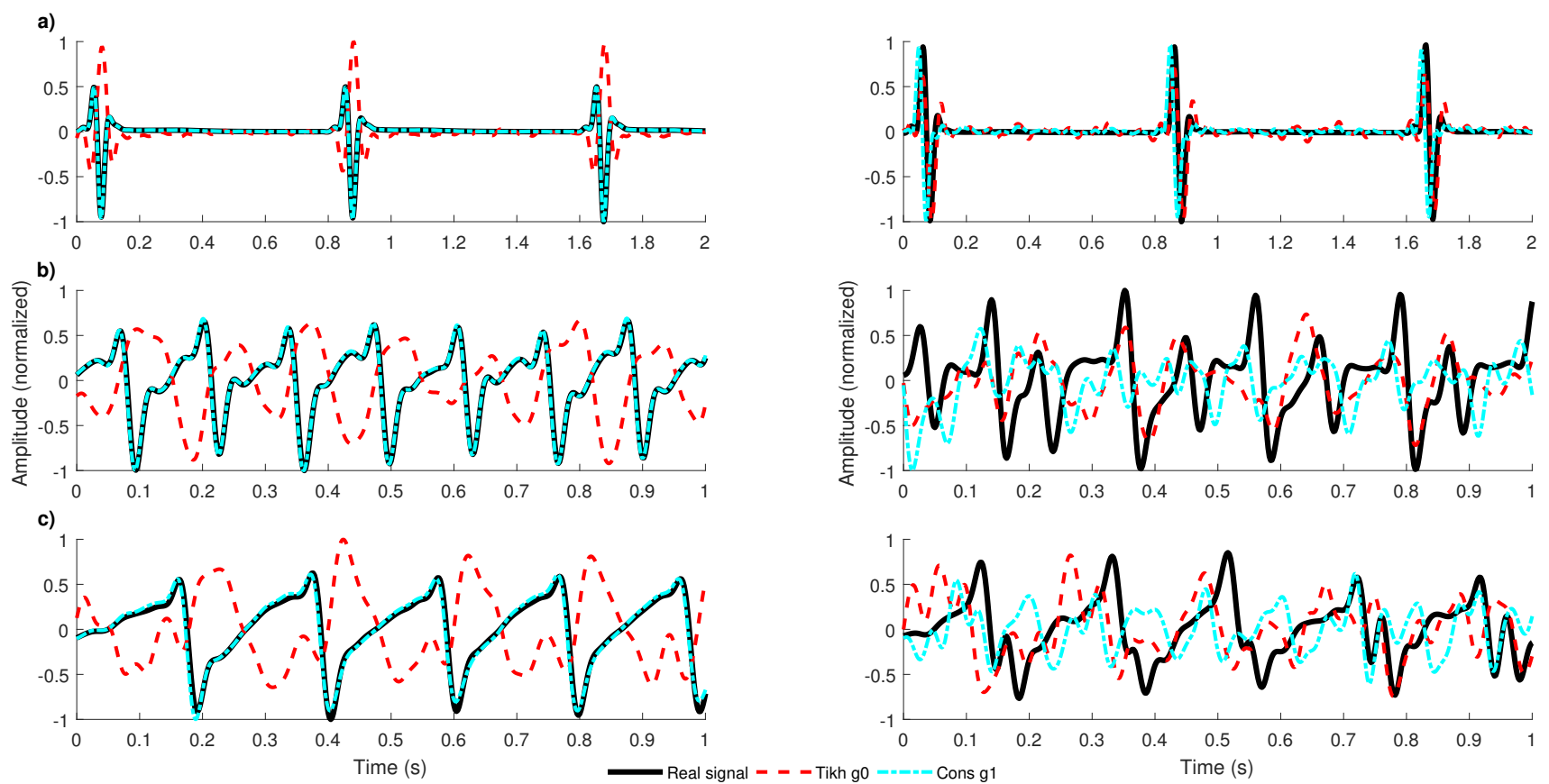

Figure 2. Comparison of real and estimated signals using Classical Tikhonov (zero-order) and Constrained Tikhonov (first-order), in SR (a), SAF (b) and CAF (c) models, in two different nodes (left and right).

of the methods were able to reconstruct correctly the epicardial potentials. However, estimations using Cons-Tikh were better than Tikh approaches.

Figure 3 shows epicardial potentials maps obtained for each model with the best Tikh and Cons-Tikh assessed methods. First-order Cons-Tikh method outperformed Tikh formulation for every model. Propagation patterns were better estimated with Cons-Tikh, both in SR and SAF model. However, performance was degraded in CAF epicardial potentials estimation, and propagation patterns are not easy to distinguish.

\section{Discussion and conclusions}

In this work, we proposed a new Tikhonov-based regularization method taking into account a priori information in the inverse problem of ECGI, by incorporating a constraint on measurements near the endocardium. The proposed model improved estimation of epicardial potentials, mainly using first-order Cons-Tikh, which favours flat, constant solutions, and penalize gradients, being able to reproduce high frequency components in the solution. Therefore, avoiding the low-pass filtering behaviour of classical Tikhonov approach.

Computing time for Cons-Tikh is higher than in Tikh methods, due to the necessity of evaluate optimal values for two parameters instead of one. However, the number of evaluated values can be lowered if we study the range of optimal parameters for a higher number of models.
Regarding regularization parameters, L-Hypersurface gives a higher weight to intracardiac measurements, and lower to BSP, but differences between models and SNR (using the same order in Tikhonov formulations) were not significant. This result was expected, as a priori information are the same intracardiac measurements used to calculate the BSP that were corrupted lately to compute the inverse problem. Therefore, one limitation of this study is the idealist environment for the experiment, and it is necessary to validate this new methodology with data obtained from $\mathrm{AF}$ patients.

\section{Acknowledgements}

This work has been partially supported by TEC201346067-R (Ministerio de Economía y Competitividad, Spanish Government).

\section{References}

[1] Figuera C, Suárez-Gutiérrez V, Hernández-Romero I, Rodrigo M, Liberos A, Atienza F, Guillem MS, BarqueroPérez Ó, Climent AM, Alonso-Atienza F. Regularization Techniques for ECG Imaging during Atrial Fibrillation: A Computational Study. Frontiers in Physiology oct 2016; 7:466. ISSN 1664-042X.

[2] Burstein B, Nattel S. Atrial fibrosis: mechanisms and clinical relevance in atrial fibrillation. J Am Coll Cardiol 2008; 51:802-809. 
Table 1. Epicardial estimation performance for SR, SAF and CAF models for SNR=20: mean RDMS, mean CC and mean RMSE with zero, first and second-order Tikhonov-based methods.

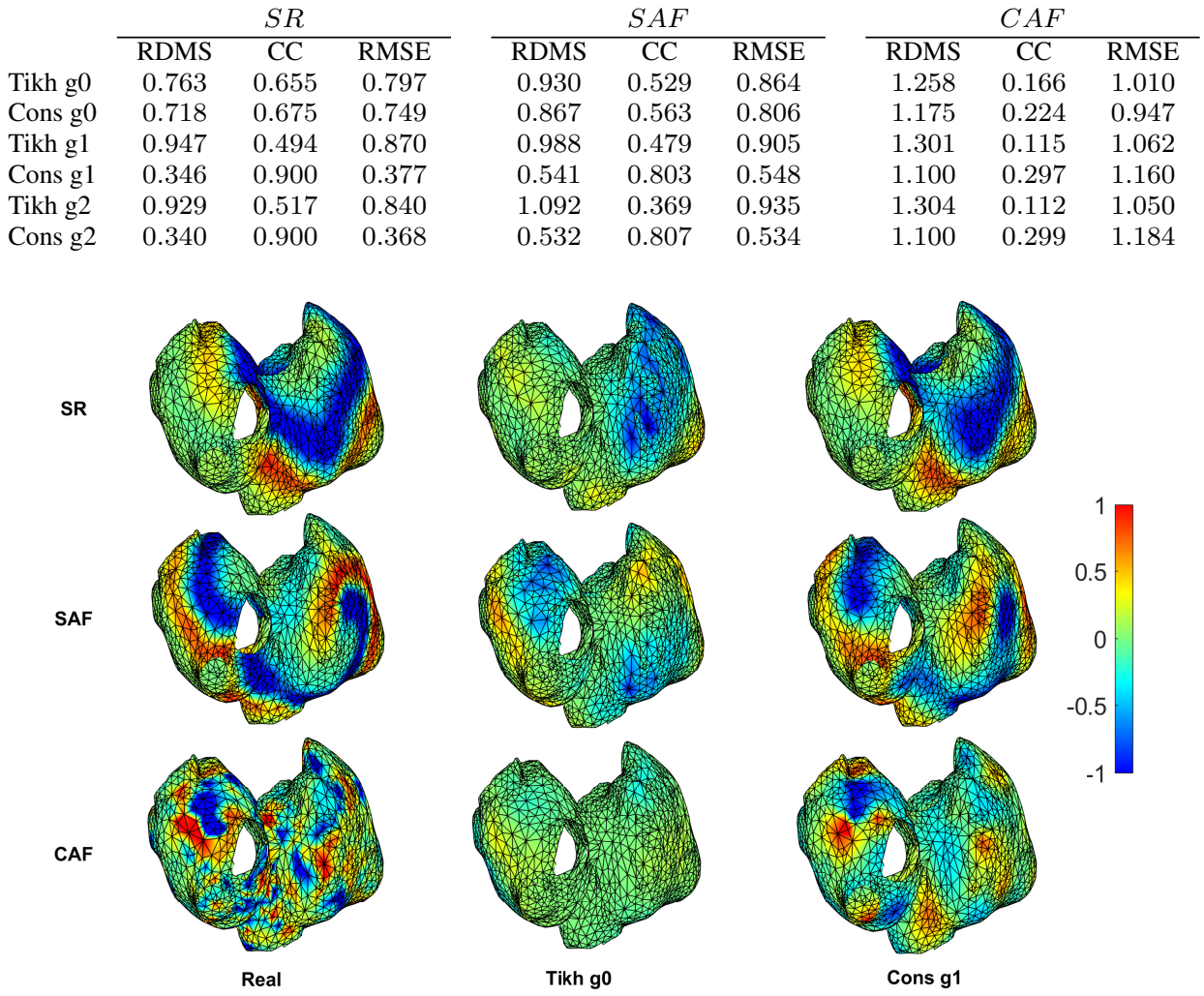

Figure 3. Comparison of real and estimated epicardial potential maps using zero-order Tikh and first-order Cons-Tikh methods, in SR, SAF and CAF models.

[3] V. Fuster, et al. Acc/aha/esc 2006 guidelines for the management of patients with atrial fibrillation: full text. Europace 2006;8(9):651-745.

[4] Guillem M, Climent A, Rodrigo M, Fernández-Avilés F, Atienza F, Berenfeld O. Presence and stability of rotors in atrial fibrillation: evidence and therapeutic implications. Cardiovascular Res 2016;109(4):480-492.

[5] Haissaguerre M, Hocini M, Shah AJ, Derval N, Sacher F, Jais P, Dubois R. Noninvasive panoramic mapping of human atrial fibrillation mechanisms: A feasibility report. Journal of Cardiovascular Electrophysiology 2013; 24(6):711-717.

[6] Rodrigo M, Climent AM, Liberos A, Calvo D, FernándezAvilés F, Berenfeld $\mathrm{O}$, et al. Identification of dominant excitation patterns and sources of atrial fibrillation by causality analysis. Annals of Biomedical Engineering 2016; 44(8):2364-2376.

[7] Brooks DH, Macleod R. Electrical imaging of the heart. IEEE Signal Process Mag 1997;14:24-42.

[8] Gulrajani R. The forward and inverse problems of electrocardiography. IEEE Eng Med Biol Sep. 1998;17(5):84101.

[9] Oster HS, Rudy Y. The use of temporal information in the regularization of the inverse problem in electrocardiography. IEEE Trans Biomed Eng 1992;39:65-75.

[10] Tikhonov AN, Arsenin VY. Solutions of Ill-posed Problems. New York: Wiley, 1977.

[11] Pedrón-Torrecilla J, Rodrigo M, Climent A, Liberos A, Pérez-David E, Bermejo J, et al. Noninvasive estimation of epicardial dominant high-frequency regions during atrial fibrillation. J Cardiovascular Electrophysiol 2016; 27(4):435-442.

[12] Belge M, Kilmer ME, Miller EL. Efficient determination of multiple regularization parameters in a generalized L-curve framework. Inverse Problems 2002;18:1161-1183.

[13] Serinagaoglu Y, Brooks DH, Macleod RS. Improved performance of bayesian solutions for inverse electrocardiography using multiple information sources. IEEE Transactions on Biomedical Engineering Oct 2006;53(10):20242034. ISSN 0018-9294.

Address for correspondence:

Carlos Figuera (carlos.figuera@urjc.es). Dept. III-D206, Camino del Molino s/n. 28943 - Fuenlabrada (Madrid), Spain. 